\title{
EXPANSIONS OF DISTRIBUTIONS
}

\author{
BY \\ GILBERT G. WALTER( $\left.{ }^{1}\right)$
}

1. Introduction. Schwartz [7] and Korevaar [4] have obtained results on the Hermite series of certain classes of distributions. Thus distributions which are finite order derivatives of functions growing no faster than $e^{c x^{2}}$, $c<\frac{1}{2}$, are uniquely determined by their Hermite series. The Hermite expansion of every distribution of polynomial growth (tempered distribution) converges to it in the distribution sense. Similarly (it is well known) distributions of period $2 \pi$ are uniquely determined by their Fourier coefficients and have Fourier series convergent to them in the distribution sense.

In this paper, expansions of distributions with respect to some general classes of complete orthonormal systems are investigated. Since a uniqueness theorem is not always available, other methods are used to prove the convergence of a series to the desired distribution. These methods are also used to get related results.

The first principal result gives a condition for the completeness of an orthonormal system on $(0,1)$, namely, that the expansion with respect to it of $\delta(x-y)$ converge to it in the distribution sense on $(0,1) \times(0,1)$. This condition is necessary and sufficient. Certain complete orthonormal systems (abbreviated C.O.N.'s) $\left\{g_{n}\right\}$ on $(0,1)$ are studied. They are those which have the property that for each integer $q \geqq 0$ there is an integer $p \geqq 0$, such that the series

$$
\sum_{n}\left\|g_{n}^{(-p)}\right\|_{\infty}\left\|g_{n}^{(q)}\right\|_{\infty}
$$

converges. Here $g_{n}^{(-p)}$ denotes an appropriate anti-derivative of order $p$ of $g_{n}$. For such C.O.N.'s, the expansion of every integrable distribution converges to it. The Legendre polynomials furnish an example of a system satisfying this condition.

Those C.O.N.'s $\left\{g_{n}\right\}$ which consist of the eigenfunctions of a regular selfadjoint boundary value problem on a finite interval $(a, b)$ are considered. If $\left\{a_{n}\right\}$ is the sequence of expansion coefficients of an integrable distribution, then there are positive constants $M$ and $m$ such that $\left|a_{n}\right|<M\left|\lambda_{n}\right|^{m}$, where $\left\{\lambda_{n}\right\}$ is the sequence of nonzero eigenvalues of the boundary value problem. On the other hand, any series $\sum a_{n} g_{n}$ whose coefficients obey this inequality

Presented to the Society, January 25, 1963 under the title Some orthogonal expansions of distributions; received by the editors July 31, 1963 and, in revised form, December 29, 1963.

(1) This research was supported in part by the National Science Foundation under Grant No. 10093 (J. Korevaar, principal investigator). 
converges to a distribution. The expansion of an integrable distribution converges to it.

Finally, a system is considered in which a uniqueness theorem can be used, that of the spherical harmonics (on the surface of the sphere). The expansion in spherical harmonics of every distribution on the sphere converges to it.

The fundamental sequence approach to distributions will be used in this paper. That is, distributions will be considered to be equivalence classes of fundamental sequences rather than functionals. This is the approach used by Mikusiński and Sikorski [5] and Korevaar [3]. Following Korevaar, the fundamental sequences will be made up, in general, of integrable functions. A distribution of order $m$ will be an $m$ th order generalized derivative of an integrable function. (This does not agree with Schwartz.)

If $\left\{f_{k}\right\}$ is a fundamental sequence belonging to the distribution $f$ on an interval $I$, and $\left\{f_{k}^{(-p)}\right\}$ converges in the mean on $I$, the first sequence will be said to be $p$-convergent to $f$ on $I$. When $p$ is not specified, the sequence $\left\{f_{k}\right\}$ will be said to converge in the sense of distributions.

In higher dimensions, the integer $p$ must be replaced by an $n$-tuple of integers. A sequence $\left\{f_{k}\right\}$ of functions integrable on an $n$-dimensional rectangle $R$ is said to be $\left(p_{1}, p_{2}, \cdots, p_{n}\right)$-convergent if there is a sequence of anti-derivatives of order $p=\left(p_{1}, p_{2}, \cdots, p_{n}\right)$ which converge in the mean on $R$. A function $g$ is called an anti-derivative of $f$ of order $p$ if $g$ can be written as an indefinite integral of order $p$ of $f$ and an integrable pseudo-polynomial of degree $<p$.

The concept of integrable distribution will be needed. A definition slightly more restrictive than that given in $[5]$ will be used, since often the behavior of the distribution outside of a finite interval $(a, b)$ will be unknown. Since each distribution has an anti-derivative distribution, the following makes sense.

Definition. A distribution $f$ is integrable from $a$ to $b$ iff there is an antiderivative distribution $g$ of $f$ having values at $a$ and $b$. The integral of $f$ from $a$ to $b$,

$$
\int_{a}^{b} f \text { or }\left(\int_{a}^{b} f(x) d x\right),
$$

is given by $g(b)-g(a)$.

In cases in which the behavior of the distribution is known only on the open interval $(a, b)$, the integral from $a^{+}$to $b^{-}$will be used. Its definition is given by replacing $a$ by $a^{+}$and $b$ by $b^{-}$in the above definition.

The notion of value of a distribution at a point used here (due to Lojasiewicz) is equivalent to that used in [5].

DEFinition. The distribution $f$ has the value $c$ at a point $x_{0}$ iff there is an integer $k \geqq 0$ and a continuous function $F$ such that $D^{k} F=f$ and

$$
\lim _{x \rightarrow x_{0}} \frac{F(x)}{\left(x-x_{0}\right)^{k}}=\frac{c}{k !} .
$$


A consequence of the definition of integrable distribution is the integration by parts formula (see $[5, \mathrm{p} .45]$ ). If $\omega$ is a sufficiently differentiable function on $[a, b]$ and $f$ a distribution with values at $a$ and $b$, then

$$
\int_{a}^{b} \omega^{\prime} f+\int_{a}^{b} \omega D f=\omega(b) f(b)-\omega(a) f(a) .
$$

2. A completeness condition. The main result in this section will be a theorem giving a condition for the completeness of an orthonormal system in $L^{2}(0,1)$. A preliminary result, dealing with the convergence of some particular series, is considered first.

2.1. The notation $f^{(-p)}$ shall mean (in this section) that $p$ th anti-derivative of $f$ which together with its first $p-1$ derivatives vanishes at the origin. Here $p$ is a positive integer, whereas $q$ and $m$ will denote non-negative integers.

Theorem. Let $\left\{g_{k}\right\}$ be a C.O.N. in $L^{2}(0,1)$, with each $g_{k}$ in $C^{m}[0,1]$, and with $q \leqq m$. Then the series

$$
\sum_{k=0}^{\infty}(-1)^{q} g_{k}^{(-p)}(x) g_{k}^{(q)}(y)
$$

is $(0, q)$-convergent on $S=(0,1) \times(0,1)$ to $\delta^{(q-p)}(x-y)$.

Let $U(t)$ be the unit step function. The proof depends on properties of the expansion of $U(x-y)$ as a function of $y$. Since the $k$ th expansion coefficient of $U(x-y)$ is $g_{k}^{(-1)}(x)$, we have

$$
\sum_{k=0}^{\infty} g_{k}^{(-1)}(x) g_{k}(y)=U(x-y),
$$

where the convergence is in the square mean on $(0,1)$ for each $x$ in $[0,1]$. That is,

$$
\int_{0}^{1}\left|U(x-y)-\sum_{k=0}^{K} g_{k}^{(-1)}(x) g_{k}(y)\right|^{2} d y \rightarrow 0
$$

as $K \rightarrow \infty$ for each $x$ in $[0,1]$. But the terms of this sequence of functions of $x$ are bounded by 1 (by Bessel's inequality), permitting us to integrate with respect to $x$ from 0 to 1 . It follows that the series in (1) converges in the square mean on $S=(0,1) \times(0,1)$, and hence in the mean. Integrating both sides of (1) $p-1$ times with respect to $x$ (from 0 to $x$ ), we get

$$
\sum_{k=0}^{\infty} g_{k}^{(-p)}(x) g_{k}(y)=\frac{(x-y)^{p-1}}{(p-1) !} U(x-y),
$$

the convergence being in the mean on $S$. By differentiating the series in (2) termwise and the right side globally $q$ times with respect to $y$, we get the desired conclusion. 
REMARK. If $p>q, \delta^{(q-p)}(x-y)$ is the function

$$
\frac{(x-y)^{p-q-1}}{(p-q-1) !} U(x-y) .
$$

The series in (2) may be differentiated termwise $r$ times (with $r \geqq p$ ) with respect to $x$. This differentiated series then becomes $(r, 0)$-convergent to $\delta^{(r-p)}(x-y)$.

2.2. By taking $r=p$ in the above statement, we have the proof of one direction of the

THEOREM. Let $\left\{g_{k}\right\}$ be an orthonormal system in $L^{2}(0,1)$. A necessary and sufficient condition that $\left\{g_{k}\right\}$ be complete is that the series

$$
\sum_{k=0}^{\infty} g_{k}(x) g_{k}(y)
$$

converge to $\delta(x-y)$ in the sense of distributions on $S$.

To prove the theorem in the other direction, we show first that the series

$$
\sum_{k=0}^{\infty} g_{k}^{(-1)}(x) g_{k}(y)
$$

converges in the mean on $S$ without the assumption of completeness. Indeed, $\sum\left|g_{k}^{(-1)}(x)\right|^{2}$ still converges by Bessel's inequality. Hence for each $x$ in $[0,1]$, the series (3) converges in the square mean to a function $F(x, y)$, square integrable with respect to $y$. But

$$
\int_{0}^{1}|F(x, y)|^{2} d y=\sum_{0}^{\infty}\left|g_{k}^{(-1)}(x)\right|^{2} \leqq \int_{0}^{1}|U(x-y)|^{2} d y
$$

indicating that $F$ is in $L^{2}(S)$. This enables one to use the same argument as in the case of the C.O.N. with $U(x-y)$ replaced by $F(x, y)$.

Differentiating (3) with respect to $x$ we get

$$
\sum_{k=0}^{\infty} g_{k}(x) g_{k}(y)=D^{(1,0)} F(x, y),
$$

where convergence is in the sense of distributions on $S((1,0)$-conv.). By hypothesis, the same series is assumed to converge to $\delta(x-y)$ on $S$ in the sense of distributions. Hence $D^{(1,0)} F(x, y)=\delta(x-y)$, implying that $F(x, y)$ $=U(x-y)+H(y)$, where $H$ is an integrable function on $(0,1)$ (since both $F$ and $U$ are).

Let $f$ be any function in $C^{\prime}[0,1]$ and vanishing at 0 and 1 . Multiplying each term of (3) by $f^{\prime}(x)$, we retain the square mean convergence on $S$. The new series converges to $\{U(x-y)+H(y)\} f^{\prime}(x)$. Integrating with respect to $x$ from 0 to 1 , we get 


$$
\sum_{k=0}^{\infty}\left\{\int_{0}^{1} g_{k}^{(-1)} f^{\prime}\right\} g_{k}(y)=\int_{0}^{1} U(x-y) f^{\prime}(x) d x+H(y) \int_{0}^{1} f^{\prime}(x) d x
$$

with convergence now in the square mean on $(0,1)$.

The coefficient integral on the left may be integrated by parts, and the integrals on the right of (5) may be evaluated to get

$$
\sum_{0}^{\infty}\left\{\int_{0}^{1} f g_{k}\right\} g_{k}=f
$$

This result can be shown to imply completeness of the C.O.N. since those functions in $C^{\prime}$ vanishing at 0 and at 1 form a dense subset of $L^{2}$. (If $h$ is an $L^{2}$ function with vanishing expansion coefficients, it follows that the integral of the product of $h$ with any $f$ vanishes. A standard argument then leads to the conclusion that $h$ vanishes almost everywhere.)

3. A class of C.O.N.'s having an interesting property. In this section we show that those C.O.N.'s satisfying a condition $\Gamma_{m}$ have the property that the expansions of integrable distributions converge to them. The condition is given in the

Definition. A C.O.N. $\left\{g_{k}\right\}$ in $L^{2}(0,1)$ satisfies condition $\Gamma_{m}$ when each $g_{k}$ is in $C^{m}[0,1]$ and when there is an integer $p>m$ and $a$ sequence $\left\{g_{k}^{(-p)}\right\}$ of anti-derivatives of order $p$ such that

converges.

$$
\sum_{k=0}^{\infty}\left\|g_{k}^{(-p)}\right\|_{\infty}\left\|g_{k}^{(m)}\right\|_{\infty}
$$

Here $g_{k}^{(-p)}$ no longer necessarily denotes the standard anti-derivative of the last section.

REMARK. A C.O.N. satisfying condition $\Gamma_{m}$ also satisfies condition $\Gamma_{q}$ for $q=0,1, \cdots, m$.

This can be shown by noting that there is a constant $M_{q}$ such that

$$
\left\|g_{k}^{(q)}\right\|_{\infty} \leqq M_{q}\left\|g_{k}^{(q+1)}\right\|_{\infty}
$$

for $q=0,1, \cdots, m-1$, and this statement can be proved by observing first that $\left\|g_{k}^{\prime}\right\|_{\infty} \rightarrow \infty$ as $k \rightarrow \infty$.

Indeed, each $g_{k}$ for $k$ large enough must have at least one zero $\alpha_{k}$ in $[0,1]$. Then since $1=\int_{0}^{1} g_{k}^{2}$, either $\frac{1}{2} \leqq \int_{0}^{\alpha_{k}} g_{k}^{2}$ or $\frac{1}{2} \leqq \int_{\alpha_{k}}^{1} g_{k}^{2}$. Assume the first possibility holds, and let $g_{k}^{(-1)}$ be such that $g_{k}^{(-1)}(0)=0$. Then, by integrating by parts, get

$$
\frac{1}{2} \leqq\left|\int_{0}^{\alpha_{k}} g_{k}^{\prime} g_{k}^{(-1)}\right| \leqq\left\|g_{k}^{\prime}\right\|_{\infty}\left\|g_{k}^{(-1)}\right\|_{2}
$$

Since termwise integration of $\sum_{k=1}^{\infty}\left(g_{k}^{(-1)}(x)\right)^{2}$ is permissible and implies that $\left\|g_{k}^{(-1)}\right\|_{2} \rightarrow 0$ as $k \rightarrow \infty$, the statement follows. 
The proof may be completed by using Taylor's formula

$$
g_{k}^{(q-1)}(x+h)=g_{k}^{(q-1)}(x)+h g_{k}^{(q)}(x)+\frac{h^{2}}{2} g_{k}^{(q+1)}(\xi)
$$

By first solving for $g_{k}^{(q)}(x)$, we get the inequality

$$
\left\|g_{k}^{(q)}\right\|_{\infty} \leqq \frac{2}{|h|}\left\|g_{k}^{(q-1)}\right\|_{\infty}+\frac{|h|}{2}\left\|g_{k}^{(q+1)}\right\|_{\infty}
$$

holding for $q=0,1, \cdots, m-1$. In particular it holds for $q=0$, whence, taking $h=4^{-m} / 2$ and noting that $\left\|g_{k}^{(-1)}\right\|_{\infty} \leqq 1$, we get

$$
\left\|g_{k}\right\|_{\infty} \leqq 4^{m+1}+4^{-m-1}\left\|g_{k}^{\prime}\right\|_{\infty} \text {. }
$$

Corresponding to $q=1$, and $h=4^{-m}$, one has

$$
\begin{aligned}
\left\|g_{k}^{\prime}\right\|_{\infty} & \leqq 2 \cdot 4^{m}\left\|g_{k}\right\|_{\infty}+2 \cdot 4^{-m-1}\left\|g_{k}^{\prime \prime}\right\|_{\infty} \\
& \leqq 2 \cdot 4^{2 m+1}+\frac{1}{2}\left\|g_{k}^{\prime}\right\|_{\infty}+2 \cdot 4^{-m-1}\left\|g_{k}^{\prime \prime}\right\|_{\infty},
\end{aligned}
$$

or

$$
\left\|g_{k}^{\prime}\right\|_{\infty} \leqq 4^{2 m+2}+4^{-m}\left\|g_{k}^{\prime \prime}\right\|_{\infty}
$$

A similar inequality can be found for $0 \leqq q \leqq m-1$ by induction. From this the desired inequality and hence the remark follows easily.

3.1. Before taking up the expansion of a distribution with respect to such C.O.N.'s, we consider the convergence of a related series.

Theorem. Let $\left\{g_{k}\right\}$ be a C.O.N. in $L^{2}(0,1)$ satisfying condition $\Gamma_{m}$. Let $f$ be a distribution given by $f=D^{m} F$ where $F$ is an integrable function on $(0,1)$ and let $a_{k}=(-1)^{m} \int_{0}^{1} F_{k}^{(m)}$. Then the series $\sum a_{k} g_{k}$ is convergent to $f$ in the sense of distributions on (0.1).

The hypothesis implies that there is a $p$ such that the series

$$
\sum_{k=0}^{\infty}(-1)^{m} g_{k}^{(-p)}(x) g_{k}^{(m)}(y)
$$

converges uniformly on $S$. Denoting the sum function by $V(x, y)$ we have

$$
D^{(p, 0)} V(x, y)=(-1)^{m} \sum_{k} g_{k}(x) g_{k}^{(m)}(y)=D^{m} \delta(x-y)
$$

since by Theorem $2.2, \delta(x-y)=\sum_{k} g_{k}(x) g_{k}(y)$. Thus, $V(x, y)$ is an antiderivative of order $(p, 0)$ of $D^{m} \delta(x-y)$, and can be written, in general, as

$$
V(x, y)=\frac{(x-y)^{p-m-1}}{(p-m-1) !} U(x-y)+x^{p-1} H_{1}(y)+x^{p-2} H_{2}(y)+\cdots+H_{p}(y),
$$

where $H_{i}(y), i=1, \cdots, p$, is a bounded integrable function on $(0,1)$ (in fact, is continuous if $p>m+1$ ). 
Multiplication of each term of (1) by $F(y)$ and integration from 0 to 1 with respect to $y$ gives us

$$
\sum_{k=0}^{\infty}(-1)^{m} g_{k}^{(-p)}(x)\left\{\int_{0}^{1} g_{k}^{(m)}(y) F(y) d y\right\}=F^{(m-p)}(x)+R_{p-1}(x),
$$

the convergence now being uniform in $x$. (Here $R_{p-1}(x)$ is a polynomial of degree $p-1$.) By differentiating each side $p$ times, we reach the conclusion of the theorem.

Quite a few examples of C.O.N.'s satisfying condition $\Gamma_{m}$ exist. One that immediately comes to mind is the trigonometric system. Another one is given in the following

Example. The Legendre polynomials $\left\{P_{n}\right\}$ satisfy the recurrence relation

$$
P_{n+1}^{\prime}(x)-P_{n-1}^{\prime}(x)=(2 n+1) P_{n}(x)
$$

for $x$ in $[-1,1]$. Using this repeatedly, one can derive the inequality which may be found, e.g., in [6],

$$
\left|P_{n}^{(m)}(x)\right| \leqq n^{2 m} / m ! .
$$

One can use the same relation to go the other way, namely

$$
\left|P_{n}^{(-1)}(x)\right|=\left|\int_{-1}^{x} P_{n}(x) d x\right|=\frac{\left|P_{n+1}(x)-P_{n-1}(x)\right|}{2 n+1}<n^{-1},
$$

and can repeat it to show

$$
\left|P_{n}^{(-m)}(x)\right|<C_{m} n^{-m}
$$

The normalized Legendre polynomials $\left\{p_{n}\right\}$ constitute a C.O.N. $\left(p_{n}(x)\right.$ $\left.=P_{n}(x)\left(n+\frac{1}{2}\right)^{1 / 2}\right)$. The series

$$
\sum_{n=m}^{\infty}\left\|p_{n}^{(-2 m-3)}\right\|_{\infty}\left\|p_{n}^{(m)}\right\|_{\infty}<C \sum_{n=m}^{\infty} n^{-2}
$$

converges, indicating that this C.O.N. satisfies the condition $\Gamma_{m}$ for any nonnegative integer $m$.

3.2. We now are ready to talk about the expansion of a distribution with respect to our C.O.N. As for a function, we define the expansion coefficients of the distribution $f$ with respect to $\left\{g_{k}\right\}$ by

$$
\int_{0^{+}}^{1^{-}} f \cdot g_{k}
$$

Notice that the integral is from $0^{+}$to $1^{-}$; if the distribution (and function) are defined on an open interval containing $[0,1]$, then we could also speak of the integral from 0 to 1 or from $0^{-}$to $1^{+}$. Of course, the integral we want does not necessarily exist for all distributions. We will limit ourselves to those for 
which it does. If each $g_{k}$ is sufficiently differentiable, then for any distribution integrable from $0^{+}$to $1^{-}$the required integrals will exist. We now give the principal

THEOREM. Let $\left\{g_{k}\right\}$ be a C.O.N. satisfying the condition $\Gamma_{m}$. Then the expansion of every distribution $f=D^{m} F$ on $(0,1)$ which is integrable from $0^{+}$to $1^{-}$ converges to $f$ (in the sense of distributions) on $(0,1)$.

The expansion coefficients of $f$ are given by

$$
c_{k}=\int_{0^{+}}^{1^{-}} f \cdot g_{k}=\int_{0^{+}}^{1^{-}} D^{m} F \cdot g_{k} .
$$

Since $D^{m} F$ is integrable from $0^{+}$to $1^{-}, D^{m-1} F$ must have a value at $0^{+}$and $1^{-}$. But if $D^{m-1} F$ has values at $0^{+}$and $1^{-}$, so does $D^{m-2} F$. In fact, all global derivatives of order less than $m$ do. Hence, repeated integration by parts (of distributions) is legitimate, i.e.,

$$
c_{k}=\left[D^{m-1} F g_{k}-D^{m-2} F g_{k}^{\prime}+\cdots+(-1)^{m-1} F g_{k}^{(m-1)}\right]_{0^{+}}^{1^{-}}+(-1)^{m} \int_{0}^{1} F g_{k}^{(m)}
$$

$$
=\sum_{q=0}^{m-1} \alpha_{q} g_{k}^{(q)}\left(0^{+}\right)+\beta_{q} g_{k}^{(q)}\left(1^{-}\right)+a_{k} .
$$

From Theorem 3.1 we know there is an integer $p$ such that

$$
\sum_{k=0}^{\infty} a_{k} g_{k}=f
$$

in the sense of $p$-convergence on $(0,1)$. Thus, in order that the expansion also converge to $f$, the series composed of the integrated terms in (2) must equal 0 . Letting $y$ be first 0 and then 1 in (1) we see that

$$
\sum_{k=0}^{\infty} g_{k}^{(-p)}(x) g_{k}^{(q)}(0)=(-1)^{q} \frac{x^{p-q-1}}{(p-q-1) !}+x^{p-1} H_{1}(0)+\cdots+H_{p}(0),
$$

and

$$
\sum_{k=0}^{\infty} g_{k}^{(-p)}(x) g_{k}^{(q)}(1)=x^{p-1} H_{1}(1)+\cdots+H_{p}(1) \quad(q=0, \cdots, m-1),
$$

where the convergence is, by hypothesis, uniform. By differentiating $p$ times, we find both $\sum_{k=0}^{\infty} g_{k}^{(q)}(0) g_{k}$ and $\sum_{k=0}^{\infty} g_{k}^{(q)}(1) g_{k}$ to be $p$-convergent to 0 on $(0,1)$. Hence

$$
\sum_{k=0}^{\infty}\left\{\sum_{q=0}^{m-1} \alpha_{q} g_{k}^{(q)}(0)+\beta_{q} g_{k}^{(q)}(1)\right\} g_{k}=0
$$

which is what was to have been shown.

If the distribution $f$ is not necessarily integrable from $0^{+}$to $1^{-}$, but is de- 
fined on the interval $J \supset[0,1]$, we can make an alternate statement in the form of the following

Corollary. Let $f=D^{m} F$, where the function $F$ is integrable over the open interval $J \supset[0,1]$, and let $\tilde{f}=D^{m} \widetilde{F}$, where $\widetilde{F}=F$ in $(0,1)$ and 0 elsewhere. Then

$$
\sum_{k=0}^{\infty}\left\{\int_{0^{-}}^{1^{+}} \tilde{f} g_{k}\right\} g_{k}=f
$$

convergence being in the sense of distributions on $(0,1)$.

As in the proof of the theorem, repeated integration by parts is permissible; it gives us

$$
\int_{0^{-}}^{1^{+}} \tilde{f} g_{k}=\int_{0^{-}}^{1^{+}} D^{m} \tilde{F} \cdot g_{k}=(-1)^{m} \int_{0}^{1} \widetilde{F} \cdot g_{k}^{(m)}=(-1)^{m} \int_{0}^{1} F \cdot g_{k}^{(m)} .
$$

This time it is simpler since $D^{q} F=0$ outside $[0,1]$ for $q=1,2, \cdots$, and all the integrated terms vanish. Hence the series $\sum_{k=0}^{\infty}\left\{\int_{0^{-}}^{1+} \tilde{f} g_{k}\right\} g_{k}$ and $\sum_{k=0}^{\infty} a_{k} g_{k}$ are identical. The latter series was shown in Theorem 3.1 to converge to $f$. The conclusion follows.

Notice that the same series also converges to $\tilde{f}$, and, in fact, to any distribution equal to $f$ in $(0,1)$.

4.C.O.N.'s of eigenfunctions. It is possible to get additional results involving the rate of growth of coefficients if the C.O.N. is made up of eigenfunctions of a self-adjoint boundary value problem.

Let $P$ be the $n$th order differential operator given by

$$
P x=p_{0} x^{(n)}+p_{1} x^{(n-1)}+\cdots+p_{n} x,
$$

where the $p_{j}(j=0,1, \cdots, n)$ are real valued functions of class $C^{\infty}$ on the closed interval $[a, b]$ and $p_{0}(x) \neq 0$ on $[a, b]$. This operator, together with appropriate boundary conditions determines a boundary value problem. We assume it to be self-adjoint and to have only nonzero eigenvalues. The characteristic pairs will be denoted by $\left(\lambda_{k}, g_{k}\right)$, and the eigenfunctions will be assumed to be normalized (and hence will form a C.O.N.). The Green's function associated with the problem will be denoted by $G(t, \tau)$ with $t$ and $\tau$ in $[a, b]$.

4.1. The results of $\$ 3$ can be shown to apply to the class of C.O.N.'s considered in this section. It is sufficient to show that property $\Gamma_{q}$ holds. That it does follows from the

Lemma. There is a constant $M_{m}$ such that

$$
\left\|g_{k}^{(m)}\right\|_{\infty} \leqq\left|\lambda_{k}\right|^{(m+1) / n} M_{m} \quad(k=0,1, \cdots ; m=0, \pm 1, \pm 2, \cdots)
$$


for the derivatives and suitable anti-derivatives of the $\boldsymbol{g}_{\boldsymbol{k}}$.

Since the integral operator $\mathscr{G}$, whose kernel is the Green's function $G$, is the inverse of $P$, the characteristic pairs obey $g_{k}=\lambda_{k} \mathscr{G}_{k}$. Hence

$$
g_{k}^{(m)}(t)=\lambda_{k} \int_{a}^{b} \frac{\partial^{m} G}{\partial t^{m}}(t, s) g_{k}(s) d s \quad(m=0,1, \cdots, n-1)
$$

since the continuity properties of the first $n-1$ derivatives of $G$ make it permissible to differentiate as much as $n-1$ times under the integral sign. Thus

$$
\left\|g_{k}^{(m)}\right\|_{\infty} \leqq\left|\lambda_{k}\right| \sup _{t \in[a, b]}\left\{\int_{a}^{b}\left|\frac{\partial^{m} G}{\partial t^{m}}(t, s)\right|^{2} d s\right\}^{1 / 2} \leqq\left|\lambda_{k}\right| M_{m} \quad(m=0, \cdots, n-1) .
$$

Let $g_{k}^{(-1)}$ denote the standard anti-derivative of $g_{k}$. Then

$$
\left\|g_{k}^{(-1)}\right\|_{\infty} \leqq \int_{a}^{b}\left|g_{k}\right| \leqq(b-a)^{1 / 2}=M_{-1} .
$$

It is possible to use the procedure in $\S 3$ to get a better bound for $\left\|g_{k}^{(m)}\right\|_{\infty}$ when $m=0,1,2, \cdots, n-2$. Indeed, the inequality used there,

$$
\left|g_{k}^{(m)}(x)\right| \leqq \frac{2}{|h|}\left\|g_{k}^{(m-1)}\right\|_{\infty}+\frac{|h|}{2}\left\|g_{k}^{(m+1)}\right\|_{\infty},
$$

is now used with $h$ taken as

$$
|h|=C \frac{\left\|g_{k}^{(m-1)}\right\|_{\infty}^{1 / 2}}{\left\|g_{k}^{(m+1)}\right\|_{\infty}^{1 / 2}}
$$

Since $\left\|g_{k}^{(m)}\right\|_{\infty} \leqq M\left\|g_{k}^{(m-1)}\right\|_{\infty}$, we can use the same $C$ for all $k$, and still permit $h$ to be sufficiently small. This gives

$$
\left\|g_{k}^{(m)}\right\|_{\infty} \leqq C_{2}\left\|g_{k}^{(m-1)}\right\|_{\infty}^{1 / 2}\left\|g_{k}^{(m+1)}\right\|_{\infty}^{1 / 2} .
$$

By substituting the same inequality with $m$ replaced by $m+1$ in the factor on the right, and solving the resulting inequality for $\left\|g_{k}^{(m)}\right\|_{\infty}$, we get

$$
\left\|g_{k}^{(m)}\right\|_{\infty} \leqq C_{3}\left\|g_{k}^{(m-1)}\right\|_{\infty}^{2 / 3}\left\|g_{k}^{(m+2)}\right\|_{\infty}^{1 / 3} .
$$

In a similar manner, one can show, for $m=0,1, \cdots, n-2$,

$$
\left\|g_{k}^{(m)}\right\|_{\infty} \leqq C_{n}\left\|g_{k}^{(-1)}\right\|_{\infty}^{(n-m-1) / n}\left\|g_{k}^{(n-1)}\right\|_{\infty}^{(m+1) / n} \leqq M_{m}\left|\lambda_{k}\right|^{(m+1) / n} .
$$

Thus the lemma holds for $m=-1,0,1, \cdots, n-1$.

The operator $P$ may now be used to extend this inequality to higher derivatives. Solving $P g_{k}=\lambda_{k} g_{k}$ for $g_{k}^{(n)}$, we get

$$
g_{k}^{(n)}=\frac{1}{p_{0}}\left(\lambda_{k} g_{k}-\sum_{i=0}^{n-1} p_{n-i} g_{k}^{(i)}\right)
$$

and hence

$$
\left\|g_{k}^{(n)}\right\|_{\infty} \leqq M_{n}\left|\lambda_{k}\right|^{(n+1) ; n}
$$


For still higher derivatives, one differentiates the right side of the equality above repeatedly.

For the anti-derivatives, a similar procedure is used. Since $\left|\lambda_{k}\right| \rightarrow \infty$, it is possible to write, for $k$ sufficiently large

$$
g_{k}=\frac{1}{\lambda_{k}-p_{n}} \sum_{i=1}^{n} p_{n-i} g_{k}^{(i)}
$$

An $m$ th anti-derivative $g_{k}^{(-m)}(m=1,2, \ldots)$ of $g_{k}$ exists such that

$$
\begin{aligned}
& g_{k}^{(-m)}=\frac{1}{\lambda_{k}} \sum_{i=1}^{n}\left(q_{n-i} g_{k}^{(i)}\right)^{(-m)} \\
&=\frac{1}{\lambda_{k}} \sum_{i=1}^{n}\left\{q_{n-i} g_{k}^{(i-m)}-\left(\begin{array}{c}
m \\
1
\end{array}\right)\left(q_{n-i}^{\prime} g_{k}^{(i-m)}\right)^{(-1)}\right. \\
&\left.+\cdots+(-1)^{m}\left(q_{n-i}^{(m)} g_{k}^{(i-m)}\right)^{(-m)}\right\} .
\end{aligned}
$$

See $\left[3\right.$, p. 494]. Here $q_{n-i}=p_{n-i} /\left(1-p_{n} / \lambda_{k}\right)$. We use this last expression with the already defined anti-derivatives of $g_{k}$ together with repeated integration from $a$ to define $g_{k}^{(-m)}$. Induction proves our inequality. (Note that $g_{k}^{(-1)}$ defined here is not the standard anti-derivative used before.)

From this property of our C.O.N., we can invoke the theorem of $\$ 3.2$ to get the

Corollary. Suppose $f$ is a distribution on $(a, b)$ which is integrable from $a^{+}$ to $b^{-}$. Then the expansion of $f$ converges to $f$ in the sense of distributions on $(a, b)$.

Take $p$ and $q$ as positive integers such that $p-q \geqq 2 n+2$. Then our C.O.N. satisfies condition $\Gamma_{q}$ (with the interval $(0,1)$ replaced by $(a, b)$ ).

4.2. We now look at the rate of growth of the coefficients in series of our C.O.N. The desired result will require some new techniques. These new techniques involve iterates $P^{m}$ of the differential operator $P$ and iterates $G_{m}$ of the Green's function $G$, some of whose properties are given in the following lemmas.

Lemma A. For each positive integer $p$, there is a number $L$ such that for each $m \geqq L$, the mth iterate $G_{m}$ of $G$ is in class $C^{p}$ on the square $S=[a, b] \times[a, b]$.

The series

$$
\sum_{k=0}^{\infty} \frac{\left|g_{k}^{(p-j)}(x) g_{k}^{(j)}(y)\right|}{\left|\lambda_{k}\right|^{m}}
$$

is majorized by

$$
M_{p-j} M_{j} \sum_{k=0}^{\infty}\left|\lambda_{k}\right|^{((p+2) / n)-m},
$$

which converges for $m \geqq L=((p+2) / n)+2$. 
Since

$$
G_{m}^{(p-j, j)}(x, y)=\sum_{k=0}^{\infty} \frac{g_{k}^{(p-j)}(x) g_{k}^{(j)}(y)}{\lambda_{k}^{m}}
$$

and since each of these functions is continuous, $G_{m}^{(p-j, j)}$ must also be continuous.

We denote by $\mathscr{G}_{m}$ the integral operator with kernel $G_{m}$. The integration by parts proves the

Corollary. If $f$ is a distribution of order $p$ on $(a, b)$ integrable from $a^{+}$to $b^{-}$, then $\mathscr{G}_{m} f$ is a function continuous on $[a, b]$.

Phrased in terms of fundamental sequences, a partial converse can be proved by using the fact that the operators $\mathscr{G}_{m}$ and $P^{m}$ are inverses. It is

Lemma B. Let $\left\{h_{k}\right\}$ be a sequence of integrable functions such that $\left\{\mathscr{G}_{m} h_{k}\right\}$ converges in the mean on $(a, b)$. Then $\left\{h_{k}\right\}$ is mn-convergent on $(a, b)$.

From the hypothesis it follows that $\left\{D^{i} \mathscr{G}_{m} h_{k}\right\}$ is $i$-convergent and hence also $j$-convergent for $j>i$. So is $\left\{p D^{i \mathscr{G}_{m}} h_{k}\right\}$ for any function $p$ in $C^{\infty}[a, b]$. So are sequences composed of sums of these. The sequence $\left\{P^{m} \mathscr{G}_{m} h_{k}\right\}=\left\{h_{k}\right\}$ is one such.

We shall need a statement similar to the above corollary, but stronger and phrased in terms of fundamental sequences also.

LEMma C. Let $\left\{f_{k}\right\}$ be a sequence of integrable functions, p-convergent on some finite interval $I$, and let $\mathscr{L}$ be a linear differential operator (with $C^{\infty}$ coefficients and zero free leading coefficient) of order $n \geqq p$. Then there is a sequence of functions $\left\{h_{k}\right\}$ such that $\mathscr{L} h_{k}=f_{k}$ for $k=1,2, \cdots$, and $\left\{h_{k}\right\}$ converges in the mean on I (uniformly if $n>p$ ).

From the theory of ordinary differential equations, one knows that the linear nonhomogeneous equation $\mathscr{L} y=f$ has a solution. Using matrix notation, and letting $W$ denote the Wronskian, $W^{-1}$ its inverse matrix, y the vector $\left(y, y^{\prime}, y^{\prime \prime}, \cdots, y^{(n-1)}\right)$, and $f$ the vector $(0,0, \cdots, 0, f)$, we see that the solution vector $\mathbf{y}$ is given by $\mathbf{y}=W \mathbf{z}$, where $D \mathbf{z}$ can be shown to be given by $W^{-1} \mathbf{f}$. Since the coefficients of $\mathscr{L}$ are assumed to be in $C^{\infty}$, the elements of $W$ are also and so are those of $W^{-1}$. (In particular, if $f \in C^{\infty}$ the equation $\mathscr{L} y=f$ has only the classical $C^{\infty}$ solutions.)

Since $\left\{f_{k}\right\}$ is $p$-convergent, so is the product $\left\{g \cdot f_{k}\right\}$ for a $C^{\infty}$ function $g$ and hence each coordinate of the vector sequence $\left\{W^{-1} \mathbf{f}_{k}\right\}$ is. But $D \mathbf{z}_{k}$ $=W^{-1} \mathbf{f}_{k}$, so each coordinate of $D z_{k}$ is a term in a $p$-convergent sequence. Taking suitable anti-derivatives, the coordinates of $\mathbf{z}_{k}$ form $(p-1)$ convergent sequences and so do those of $\mathbf{y}_{k}=W \mathbf{z}_{k}$. In particular, the last coordinate of $\mathbf{y}_{k}$ forms a $(p-1)$-convergent sequence, i.e., $\left\{y_{k}^{(n-1)}\right\}$ is $(p-1)$ convergent. Hence $\left\{y_{k}^{(n)}\right\}$ is $p$-convergent. But $p \leqq n$ by hypothesis, so that 
$\left\{y_{k}^{(n)}\right\}$ is $n$-convergent. This implies that $\left\{y_{k}\right\}$ converges in the mean on $(a, b)$ since it is already $(p-1)$-convergent. If $p<n$, the convergence is uniform, since then $\left\{y_{k}^{\prime}\right\}$ converges in the mean.

We now can prove more easily the main result of this section.

TheOREM. The partial sums of the series $\sum_{0}^{\infty} a_{k} g_{k}$ form a sequence convergent in the distribution sense on $(a, b)$ if and only if the coefficients obey the inequality

$$
\left|a_{k}\right|<M\left|\lambda_{k}\right|^{m} \quad(k=0,1,2, \cdots)
$$

for some constant $M$ and integer $m$.

Suppose first of all that the coefficients obey the inequality. Applying the operator $\mathscr{G}_{m+1}$ to the $K$ th partial sums of the series, we get

$$
\mathscr{G}_{m+1} \sum_{k=0}^{K} a_{k} g_{k}=\sum_{k=0}^{K} a_{k} \mathscr{G}_{m+1} g_{k}=\sum_{k=0}^{K} a_{k} \frac{g_{k}}{\lambda_{k}^{m+1}} .
$$

The coefficients of the series $\sum a_{k} g_{k} / \lambda_{k}^{m+1}$ are majorized by $M /\left|\lambda_{k}\right|$, hence this series converges in the mean on $(a, b)$. Thus the sequence $\left\{\mathscr{G}_{m+1} S_{K}\right\}$ converges in the mean, and by Lemma $B$, the sequence $\left\{S_{K}\right\}$ of partial sums of the series is $(m+1) n$-convergent on $(a, b)$.

To prove the theorem in the other direction, we take $\left\{S_{K}\right\}$ to be $p$ convergent for some integer $p \geqq 0$. Then by Lemma $C$, there is a sequence of functions $\left\{h_{K}\right\}$ such that $P^{m} h_{K}=S_{K}$, where $m>p / n$, which converges uniformly on $[a, b]$. Since $P^{m} g_{k}=\lambda_{k}^{m} g_{k}$, the solution of $P^{m} h_{K}=S_{K}$ is given by

$$
h_{K}=\sum_{k=0}^{K} a_{k} \frac{g_{k}}{\lambda_{k}^{m}}+R_{K} \quad(K=0,1, \ldots),
$$

where $R_{K}$ is a solution of the homogeneous equation $P^{m} y=0$.

The "nice part" of this solution can be expressed as

$$
\begin{aligned}
\sum_{k=0}^{K} a_{k} \frac{g_{k}}{\lambda_{k}^{m}} & =\sum_{k=1}^{K} \frac{S_{k}-S_{k-1}}{\lambda_{k}^{m}}+\frac{a_{0} g_{0}}{\lambda_{0}^{m}} \\
& =\sum_{k=0}^{K} \frac{S_{k}}{\lambda_{k}^{m}}-\sum_{k=0}^{K-1} \frac{S_{k}}{\lambda_{k+1}^{m}} .
\end{aligned}
$$

Since $\left\{S_{K}\right\}$ is $p$-convergent on $(a, b)$, some sequence of anti-derivatives $\left\{S_{K}^{(-p-1)}\right\}$ converges uniformly on $[a, b]$, and is therefore uniformly bounded. The integer $m$ may be taken to be greater than 2 without loss of generality, so that $\sum 1 /\left|\lambda_{k}\right|^{m}$ converges. Hence the series

$$
\sum_{k=0}^{\infty} \frac{S_{k}^{(-p-1)}}{\lambda_{k}^{m}} \text { and } \sum_{k=0}^{\infty} \frac{S_{k}^{(-p-1)}}{\lambda_{k+1}^{m}}
$$

both converge uniformly and the "nice part" sequence is $(p+1)$-convergent. Since $\left\{h_{K}\right\}$ is uniformly convergent and hence also $(p+1)$-convergent, the 
difference sequence of these two must be $(p+1)$-convergent. The difference sequence is $\left\{R_{K}\right\}$.

Now $R_{K}$, as a solution of $P^{m} y=0$, may be written as

$$
R_{K}=\sum_{i=1}^{m \cdot n} c_{i}^{K} w_{i}
$$

where $\left\{w_{i}\right\}$ is a set of $m \cdot n$ linearly independent solutions. Since fixed antiderivatives $w_{i}^{(-p-1)}$ together with $p+1$ linearly independent polynomials $Q_{j}$ of degree $\leqq p$ form a set of $(m \cdot n)+p+1$ linearly independent functions, the mean convergence of the sequence

$$
\left\{\sum_{i=0}^{m \cdot n} c_{i}^{K} w_{i}^{(-p-1)}+\sum_{j=0}^{p} b_{j}^{K} Q_{j}\right\}
$$

as $K \rightarrow \infty$ implies the convergence of the coefficient sequences. Hence the sequence

$$
\left\{\sum_{i=0}^{m \cdot n} c_{i}^{K} w_{i}=R_{K}\right\}
$$

converges uniformly.

This permits the conclusion that

$$
\sum_{k=0}^{\infty} a_{k} \frac{g_{k}}{\lambda_{k}^{m}}=\lim _{K \rightarrow \infty} h_{K}-\lim _{K \rightarrow \infty} R_{K}
$$

where the convergence is uniform on $[a, b]$. Since it is uniform it is also in the square mean on $(a, b)$, and the coefficients are bounded. That is,

$$
\left|a_{k}\right| \leqq C\left|\lambda_{k}\right|^{m} \quad(k=0,1, \cdots)
$$

and the proof is complete.

REMARK. We might have modified the last step of the proof. If we had had only a sub-interval $(\alpha, \beta)$ of $(a, b)$ every step would have been valid except the last one. Some other argument would have been needed to show that the coefficients of a uniformly convergent series on $(\alpha, \beta)$ are bounded. Since this condition is a property of certain C.O.N.'s, e.g., the trigonometric functions, we see that we have also proved the following:

If $\left\{g_{k}\right\}$ has the property that every locally uniformly convergent series in $g_{k}$ has bounded coefficients, then convergence of the series $\sum a_{k} g_{k}$ in the distribution sense on an arbitrary subinterval $(\alpha, \beta)$ of $(a, b)$ implies that there is a constant $M$ and integer $m \geqq 0$ such that

$$
\left|a_{k}\right|<M\left|\lambda_{k}\right|^{m}
$$

4.3. We now consider further results which can be proved by the methods of $\$ 4.2$. The corollary in $\$ 4.1$ was one which could have been. It was

The expansion of a distribution $f$ of finite order on $(a, b)$ which is integrable 
from $a^{+}$to $b^{-}$is convergent to the distribution on $(a, b)$ in the sense of distributions.

To prove it, we note that by the corollary to Lemma $A$ in $\$ 4.2$ there is an integer $m$ such that $F=\mathscr{G}_{m} f$ is a continuous function, and

$$
F=\sum_{k=0}^{\infty}\left\langle F, g_{k}\right\rangle g_{k}
$$

in the sense of $L^{2}(a, b)$. Hence

$$
f=\sum_{k=0}^{\infty}\left\langle F, g_{k}\right\rangle P^{m} g_{k}=\sum_{k=0}^{\infty}\left\langle F, g_{k}\right\rangle \lambda_{k}^{m} g_{k}
$$

in the sense of $m n$-convergence. But since $f$ is integrable on $\left(a^{+}, b^{-}\right)$, we have

$$
\left\langle\mathscr{G}_{m} f, g_{k}\right\rangle=\left\langle f, \mathscr{G}_{m} g_{k}\right\rangle=\frac{\left\langle f, g_{k}\right\rangle}{\lambda_{k}^{m}} \quad(k=0,1,2, \cdots) .
$$

because $\mathscr{G}_{m}$ is self-adjoint. Hence the coefficients in the series are the expansion coefficients of $f$, proving the result.

What about the case in which the distribution is not necessarily integrable on $\left(a^{+}, b^{-}\right)$? By Lemma C of $\$ 4.2$, we see that it is still possible to write $f=P^{m} F$ with the difference that $F$ now is not given by $\mathscr{G}_{m} f$ necessarily. Thus the best we can do is conclude that

$$
f=\sum_{k=0}^{\infty}\left\langle F, g_{k}\right\rangle \lambda_{k}^{m} g_{k}
$$

convergence being in the sense of distributions. Notice that other series of this kind converge to $f$. They differ by coefficients of solutions of $P^{m} y=0$. This can be shown to give us a substitute for a uniqueness theorem, namely

TheOREM. The series $\sum c_{k} g_{k}$ is convergent to 0 in the sense of distributions on $(a, b)$ if and only if there is an integer $m \geqq 0$ such that $c_{k}=\left\langle f, g_{k}\right\rangle \lambda_{k}^{m}$ for $k=0,1, \cdots$, where $f$ is a solution of $P^{m} y=0$.

Suppose $c_{k}=\left\langle f, g_{k}\right\rangle \lambda_{k}^{m}$, where $P^{m} f=0$. Then by the above remark

$$
\sum_{k=0}^{\infty} c_{k} g_{k}=\sum_{k=0}^{\infty}\left\langle f, g_{k}\right\rangle \lambda_{k}^{m} g_{k}=P^{m} f=0
$$

where the series converge in the sense of distributions on $(a, b)$.

On the other hand, if $\sum c_{k} g_{k}$ is $p$-convergent, then by the theorem of $\$ 4.2$, $\left|c_{k}\right|<M_{m-2}\left|\lambda_{k}\right|^{m-1}$ for some $m$ and all $k$. Hence the sequence

$$
\left\{\mathscr{G}_{m} \sum_{k=0}^{K} c_{k} g_{k}\right\}=\left\{\sum_{k=0}^{K} \frac{c_{k}}{\lambda_{k}^{m}} g_{k}\right\}
$$

converges in the mean to a function $F$, and the series $\sum c_{k} g_{k}$ converges to $P^{m} F$ in the sense of distributions. Since by hypothesis it equals 0 also, $P^{m} F$ $=0$. All solutions of this equation are $C^{\infty}$ functions. Thus the $k$ th expansion 
coefficient of $F$ must be given by $c_{k} / \lambda_{k}^{m}$ whence

$$
c_{k}=\lambda_{k}^{m}\left\langle F, g_{k}\right\rangle \quad(k=0,1,2, \cdots),
$$

proving the theorem.

An alternate form of this theorem, which perhaps indicates better what we have, comes from an easy integration by parts. It is the following

COROLLARY. If $\sum_{k=0}^{\infty} c_{k} g_{k}$ converges to the zero distribution on $(a, b)$, then there are constants $\alpha_{q}$ and $\beta_{q}$ such that

$$
c_{k}=\sum_{q=0}^{m n-1}\left\{\alpha_{q} g_{k}^{(q)}(a)+\beta_{q} g_{k}^{(q)}(b)\right\} \quad(k=0,1,2, \cdots) .
$$

From the theorem, we have

$$
\begin{aligned}
c_{k} & =\lambda_{k}^{m}\left\langle F, g_{k}\right\rangle=\left\langle F, \lambda_{k}^{m} g_{k}\right\rangle \\
& =\left\langle F, P^{m} g_{k}\right\rangle= \pm\left\langle P^{m} F, g_{k}\right\rangle+\text { (integrated terms). }
\end{aligned}
$$

But $P^{m} F=0$; and the general form of the integrated terms is given by the conclusion.

REMARK. A different substitute for a uniqueness theorem could be

There is at most one admissible series convergent to an integrable distribution.

(An admissible sequence $\left\{f_{k}\right\}$ is one for which $\lim _{k \rightarrow \infty} \int_{a}^{b} f_{k}=\int_{a^{+}}^{b-} f$.) Suppose the partial sums of $\sum b_{k} g_{k}$ converge to $f$ and form a sequence admissible on $\left(a^{+}, b^{-}\right)$. Then

$$
\lim _{K \rightarrow \infty} \sum_{k=0}^{K} b_{k}\left\langle g_{k}, g\right\rangle=\langle f, g\rangle
$$

whenever $g$ is sufficiently differentiable. Taking $g=g_{n}$, we have $b_{n}=\left\langle f, g_{n}\right\rangle$.

5. The spherical harmonics. The method used to get results about the trigonometric Fourier series of distributions can be used to get similar results about the expansion in spherical harmonics (the Laplace series) of a distribution on the surface of the sphere. In both cases the distribution may be considered to be defined on a compact differentiable manifold. A distribution defined on such a manifold (by means of sequences of integrable functions locally $p$-convergent) is always integrabie over it. This can be shown by the use of a differentiable partition of unity (just as with functions).

We use the notation of [6] for spherical harmonics. The expansion of an integrable function $F$ on the surface of the sphere $\Omega$ is given by

$$
F \sim \sum_{n=0}^{\infty} \frac{1}{2} a_{n 0} u_{n 0}+\sum_{m=1}^{n} a_{n m} u_{n m}+b_{n m} v_{n m}=\sum_{n=0}^{\infty} Y_{n}(F) .
$$

The $n$th spherical harmonic $Y_{n}$ may be given directly at the point $P$ on $\Omega$ by

$$
Y_{n}(F, P)=\frac{n+1 / 2}{2 \pi} \int_{\Omega} F(Q) P_{n}(\cos (P, Q)) d \Omega(Q),
$$


where $(P, Q)$ is the angular distance from $P$ to $Q ; P_{n}$, the Legendre polynomial of degree $n$.

Let $\Delta$ indicate the Laplacian operator on the sphere. Applied to any spherical harmonic $Y_{n}$, it gives

$$
\Delta Y_{n}=-n(n+1) Y_{n} .
$$

This holds in particular for the functions $v_{n m}$ or $u_{n m}$.

Green's identity enables us to show that

$$
Y_{n}(\Delta F)=\Delta Y_{n}(F)
$$

for $F \in C^{2}$. Indeed, the relation

$$
\left\langle\Delta F, u_{m n}\right\rangle=\left\langle F, \Delta u_{m n}\right\rangle=-n(n+1)\left\langle F, u_{m n}\right\rangle
$$

holds. The same one holds if $u_{m n}$ is replaced by $v_{m n}$. Hence we have $Y_{n}(\Delta F)$ $=-n(n+1) Y_{n}(F)$.

Now let $F$ be a function in $L^{1}(\Omega)$, whose integral over the sphere $\Omega$ is 0 . Then, using (2),

$$
\left|Y_{n}(F, P)\right| \leqq \frac{n+1 / 2}{2 \pi}\left\|P_{n}\right\|_{\infty} \int_{\Omega}|F(Q)| d \Omega \leqq \frac{n+1 / 2}{2 \pi} K_{1} \quad(n=0,1,2, \ldots) .
$$

Consider the series consisting of the functions $\widetilde{Y}_{n}=-Y_{n}(F) /(n+1) n$. The series $\sum\left\|\widetilde{Y}_{n}\right\|_{2}^{2}$ converges, whence the series of orthogonal functions $\sum \widetilde{Y}_{n}$ converges in the mean to a function $G$. Hence

$$
\sum \Delta \widetilde{Y}_{n}=\sum Y_{n}(F)=\Delta G
$$

in the distribution sense. We would like to show that $\Delta G=F$.

Integration by parts enables us to define spherical harmonics for distributions. Then the relation (4) can be extended to distributions (by going to fundamental sequences). Hence, since $Y_{n}(G)=\widetilde{Y}_{n}$,

$$
Y_{n}(\Delta G)=\Delta Y_{n}(G)=-n(n+1) \widetilde{Y}_{n}=Y_{n}(F) \text {. }
$$

Thus the distribution (or function) $\Delta G$ has the same spherical harmonics as the function $F$. A uniqueness theorem would enable us to conclude that $\Delta G$ equals $F$.

Theorem (Uniqueness). Let $f=\Delta^{p} H, H \in L^{2}(\Omega), p \geqq 1$, such that $Y_{n}(f)$ $=0$ for $n=0,1,2, \ldots$. Then $f=0$.

If $H \in L^{2}(\Omega)$, the fact that $Y_{n}\left(\Delta^{p} H\right)=0$ implies that $Y_{n}(H)=0$ for $n=1$, $2, \cdots$, by repeated application of (4). But the completeness ensures that if $Y_{n}(H)=0$ for $n=1,2, \cdots$, then $H$ is a constant and hence $\Delta^{p} H=0$.

Corollary. The Laplace series of an integrable function $F$ on the sphere $\Omega$ converges to $F$ in the distribution sense.

In order to show that the Laplace series of a distribution $f$ converges we 
need a bound on the spherical harmonics of $f$.

Suppose $f$ is given by the fundamental sequence $\left\{f_{k}\right\}$ of order $\left(p_{i}, q_{i}\right)$ in the neighborhood $N_{i}, i=1,2, \cdots, j\left(\bigcup_{i=1}^{j} N_{i}=\Omega\right)$. Then the coefficient $a_{m n}(f)$ is given by (the normalizing factor $((n-m) ! /(n+m) !) \cdot((2 n+1) / 2 \pi)$ times):

$$
\begin{aligned}
\int_{\Omega} f u_{n m} & =\lim _{k \rightarrow \infty} \int_{\Omega} f_{k} u_{n m} \\
& =\sum_{i=1}^{j} \int_{N_{i}} F_{i}\left(h_{i} u_{n m}\right)^{\left(p_{i}, q_{i}\right)},
\end{aligned}
$$

where $F_{i}=\lim _{k \rightarrow \infty} f_{k}^{\left(-p_{i},-q_{i}\right)}$ on $N_{i}$ and $\left\{h_{i}\right\}$ is a partition of unity with $h_{i} \equiv 0$ outside $N_{i}$. The fact that $\left|P_{n}^{(m)}(x)\right| \leqq n^{2 m} / m$ ! (see 82) enables us to conclude that $\left\|\left(h_{i} u_{n m}\right)^{\left(p_{i}, q_{i}\right)}\right\|_{\infty} \leqq C_{i} n^{v_{i}}$ for some integer $v_{i}$ and constant $C_{i}$ in $N_{i}$. Hence $a_{m n}(f)$ obeys the same sort of inequality and so does $Y_{n}(f)$, which may be written as $\left\|Y_{n}(f)\right\|_{\infty} \leqq \operatorname{Cn}^{p}(n+1)^{p} \quad(n=1,2, \ldots)$. Since $Y_{n}\left(f_{k}\right)$ $\rightarrow Y_{n}(f)$ as $k \rightarrow \infty$, the same inequality holds for $Y_{n}\left(f_{k}\right)$, uniformly in $k$.

We can now show $\sum Y_{n}(f)$ converges to a distribution. Indeed,

$$
\sum_{n \neq 0} \frac{Y_{n}(f)}{[n(n+1)]^{p+1}}
$$

converges in the mean to a function $F$ in $L^{2}(\Omega)$. Hence

$$
\sum_{n \neq 0} Y_{n}(f)=\Delta^{p+1} F
$$

in the sense of distributions. If $\tilde{f}=\Delta^{p+1} F+Y_{0}(f)$, by showing that $f=\tilde{f}$, we can prove the

ThEOREM. The Laplace series of a distribution on the sphere converges to it.

We use the uniqueness theorem to complete the proof. Indeed, define $F_{k}$ by

$$
\sum_{n \neq 0} \frac{Y_{n}\left(f_{k}\right)}{[n(n+1)]^{p+1}} .
$$

Then

$$
f_{k}-Y_{0}\left(f_{k}\right)=\Delta^{p+1} F_{k}
$$

by the uniqueness theorem. But $F_{k} \rightarrow F$ in the mean. Hence $\Delta^{p+1} F_{k} \rightarrow \Delta^{p+1} F$ in the sense of distributions. Since $Y_{0}\left(f_{k}\right) \rightarrow Y_{0}(f)$, we have $f_{k} \rightarrow \Delta^{p+1} F+Y_{0}(f)$. Thus $f=\Delta^{p+1} F+Y_{0}(f)$.

\section{BIBLIOGRAPHY}

1. E. A. Coddington and N. Levinson, Theory of ordinary differential equations, McGraw-Hill, New York, 1955.

2. D. Jackson, Fourier series and orthogonal polynomials, Carus Monograph Series No. 6, 
Mathematical Association of America, Buffalo, N. Y., 1941.

3. J. Korevaar, Distributions defined by fundamental sequences. I, II, III, IV, V, Nederl. Akad. Wetensch. Proc. Ser. A 58 (1955) = Indag. Math. 17 (1955), 368-378, 379-389, 483-493, 494-503, 663-674.

4. _ Pansions and the theory of Fourier transforms, Trans. Amer. Math. Soc. 91 (1959), 53-101.

5. J. Mikusinski and R. Sikorski, The elementary theory of distributions. I, II, Rozprawy Mat. 12 (1957); ibid. 25 (1961).

6. G. Sansone, Orthogonal functions, revised English ed., Pure and Applied Mathematics, Vol. IX, Interscience, New York, 1959.

7. L. Schwartz, Théorie des distributions, Tomes I, II, Actualités Sci. Ind. Nos.1091, 1122, Hermann, Paris, 1950, 1951.

8. E. C. Titchmarsh, Eigenfunction expansions associated with second-order differential equations, Vols. I, II, Clarendon Press, Oxford, 1946, 1958.

9. F. G. Tricomi, Integral equations, Pure and Applied Mathematics, Vol. V, Interscience, New York, 1957.

\author{
UNIVERSITY OF WisCONSIN-MiLWAUKEE \\ MiLWAUKEe, Wisconsin
}

